\title{
Correlation between fruit weight and nutritional metabolism during development in CPPU-treated Actinidia chinensis 'Hongyang'
}

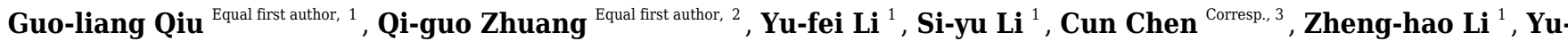 \\ yang Zhao ${ }^{3}$, Yi Yang ${ }^{1}$, Zhi-bin Liu ${ }^{\text {Corresp. } 1}$ \\ ${ }^{1}$ Key Laboratory of Bio-Resources and Eco-Environment of Ministry of Education, College of Life Sciences, Sichuan University, Chengdu, China \\ 2 Kiwifruit Breeding and Utilization Key Laboratory, Sichuan Provincial Academy of Natural Resource Sciences, Chengdu, China \\ 3 Institute of Microbiology, College of Chemistry and Life Science, Chengdu Normal University, Chengdu, China \\ Corresponding Authors: Cun Chen, Zhi-bin Liu \\ Email address: chencun@cdnu.edu.cn, liuzhibin@scu.edu.cn
}

Forchlorfenuron, N-(2-chloro-4-pyridyl)-N-phenylurea (CPPU), is often used to promote fruit growth and improve production. The role of CPPU in kiwifruit growth has been established. However, the correlation between fruit weight and nutritional metabolism during development after CPPU treatments remains largely undetermined. Here, we surveyed the variations in weight and nutrient components of the 'Hongyang' kiwifruit (Actinidia chinensis) when CPPU was sprayed on fruit 25 days after anthesis. The CPPU application did not significantly influence the dry matter, soluble solids, starch, Vitamin C or protein concentrations. However, the fresh weight, length and maximum diameter were significantly increased compared with the control. Moreover, in fruit of the same developmental stage, the fructose, glucose and soluble sugar levels increased after the CPPU treatment, compared with the control. On the contrary, citric, quinic and titratable acid concentrations decreased. However, a correlation analysis between fresh weight and the nutritional contents revealed that CPPU did not affect the concentrations of the most abundant organic acids (quinic and citric) and sugars (glucose, fructose and sucrose), compared with control fruit of the same weight. Therefore, CPPU applications enhance 'Hongyang' kiwifruit weight/size. However, there were no significant differences in the nutritional qualities of treated and untreated fruit having the same weights. 


\section{Title}

2 Correlation between fruit weight and nutritional metabolism during development in CPPU-

\section{treated Actinidia chinensis 'Hongyang'}

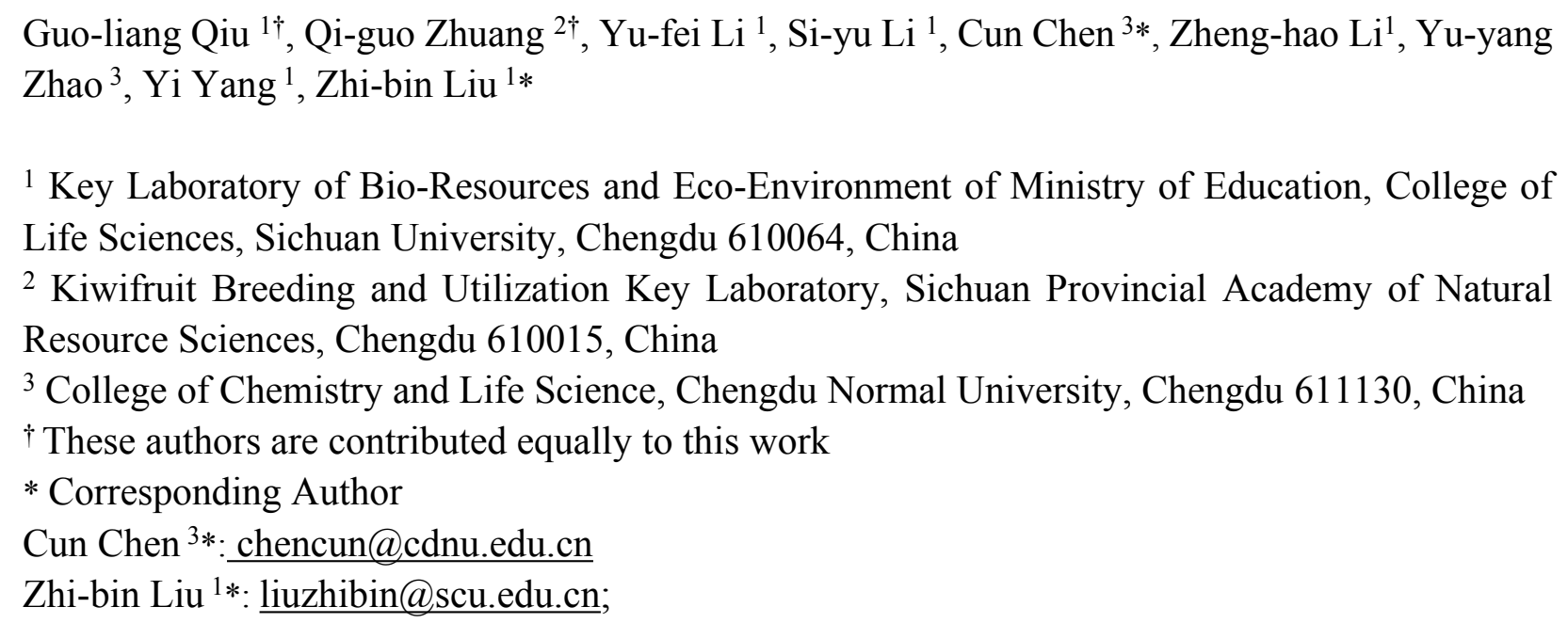

\section{Abstract}

Forchlorfenuron, N-(2-chloro-4-pyridyl)-N-phenylurea (CPPU), is often used to promote fruit growth and improve production. The role of CPPU in kiwifruit growth has been established. However, the correlation between fruit weight and nutritional metabolism during development after CPPU treatments remains largely undetermined. Here, we surveyed the variations in weight and nutrient components of the 'Hongyang' kiwifruit (Actinidia chinensis) when CPPU was sprayed on fruit 25 days after anthesis. The CPPU application did not significantly influence the dry matter, soluble solids, starch, Vitamin C or protein concentrations. However, the fresh weight, length and maximum diameter were significantly increased compared with the control. Moreover, in fruit of the same developmental stage, the fructose, glucose and soluble sugar levels increased after the CPPU treatment, compared with the control. On the contrary, citric, quinic and titratable acid concentrations decreased. However, a correlation analysis between fresh weight and the nutritional contents revealed that CPPU did not affect the concentrations of the most abundant organic acids (quinic and citric) and sugars (glucose, fructose and sucrose), compared with control fruit of the same weight. Therefore, CPPU applications enhance 'Hongyang' kiwifruit weight/size. However, there were no significant differences in the nutritional qualities of treated and untreated fruit having the same weights. 


\section{Introduction}

Owing to its pleasant fragrance, and high carbohydrate, vitamin and folic acid concentrations, the kiwifruit has become an economically and nutritionally important fruit crop (Richardson et al., 2018). China is the largest producer of kiwifruit worldwide with a production $\sim 2.04$ million tons in 2018 (FAO stat, 2018). The green-fleshed 'Hayward' (Actinidia deliciosa (A. Chev.) C.F. Liang and A.R. Ferguson.) bred in New Zealand, was the first globally traded kiwifruit cultivar. Subsequently, the yellow-fleshed 'Hort16A' (A. chinensis Planch. var. chinensis) cultivar was selected and stimulated the development of the world kiwifruit industry. The success of 'Hort16A' kiwifruit is ascribed largely to the tropical-like flavours and sweeter yellow-flesh compared with 'Hayward' kiwifruit (Pranamornkith et al., 2012; Du et al., 2019). In recent years, consumers have preferentially selected kiwifruit based on the flesh colour. 'Hongyang' (A. chinensis Planch. var. chinensis), cultivated by the Sichuan Provincial Nature Resources Institute, is the first red-fleshed kiwifruit cultivar to be grown on a commercial scale (Zhang et al., 2018). 'Hongyang' is quite popular among consumers owing to its red inner pericarp and superior fruit properties, including better taste and higher Vitamin C concentrations (He et al., 2018). Therefore, it quickly became a significant component of the Chinese kiwifruit industry.

To achieve the most desirable characteristics at harvest time, kiwifruit must attain a large wellshaped form and contain a balance of soluble sugars and organic acids (Cruz-Castillo et al., 2014). These fruit-related parameters are considered to be important in terms of quality and consumer appeal (Ainalidou et al., 2015). Therefore, fruit weight/size, and the soluble sugar and organic acid concentrations are the main parameters used to grade kiwifruit. Bioregulators, such as forchlorfenuron, N-(2-chloro-4-pyridyl)-N-phenylurea (CPPU), gibberellin, 2, 4dichlorophenoxyacetic acid (2, 4-D) and thidiazuron, are often applied to improve productivity of fruit crops (Famiani et al., 2007). Recently, the synthetic cytokinin CPPU has become the most widely used owing to a strong ability to stimulate fruit growth (Cruz-Castillo et al., 2014).

At present, it is legal to use CPPU commercially for fruit production in Italy, Japan, Chile and the USA (Cruz-Castillo et al., 2014). CPPU can significantly promote cell division and enhance the sizes of some fruit, such as watermelons, apples, grapes and macadamia. However, CPPU's effects on the fruit quality attributes were inconsistent. For example, compared with the untreated control, there were similar soluble solids concentration (SSC) and decreased total organic acid concentrations in CPPU-treated 'Cuiguan' pear (Niu et al., 2015). By contrast, CPPU applications reduced the SSC and enhanced the accumulation of titratable acidity (TA) in 'Flame Seedless' grapes (Peppi and Fidelibus, 2008). Nevertheless, Stern et al. (2003) found that CPPU applications did not produce any negative effects on the fruit quality attributes of 'Royal Gala' apple at harvest.

For kiwifruit, the SSC and TA level in CPPU-treated fruit of A. arguta (Sieb. et Zucc.) Planch. ex Miq. 'Mitsuko' were lower than those of untreated fruit (Kim et al., 2006).On the contrary, CPPU applications led to an increased SSC level and a similar TA concentration, when compared with the untreated 'Hayward' kiwifruit (Ainalidou et al., 2015). The reason for the different effects of CPPU on the fruit quality attributes of 'Mitsuko' and 'Hayward' remains undetermined. In the previous studies, the effects of CPPU on fruit nutrition were mainly focused on the same 
development stage. There was a lot of difference of fruit quality between CPPU-treated and control fruit at this point. However, the reason was mainly because the weight of CPPU-treated fruit was much more than that of control group at the same time. In our present research, we want to know whether the nutritional metabolism of fruit was affected by CPPU application at the same weight. In this study, we examined the weight and composition of 'Hongyang' at various developmental points after CPPU was applied. Then, the correlations between fruit weight and the major organic acid and sugar metabolic profiles were analysed.

\section{Materials \& Methods}

\section{Field experiment}

Field experiments were carried out during 2018 in Shifang County, Sichuan $\left(31^{\circ} 13^{\prime} \mathrm{N}\right.$, $101^{\circ} 01.161 ' \mathrm{E}, 662 \mathrm{~m}$ a.s.1.). The grafted kiwifruit vines were trained by a Pergola system with 3$\mathrm{m}$ spacings within rows and 4-m spacings between rows. In total, 40 representative vines were randomly selected and labelled for the subsequent experiments. At 25 days after anthesis (DAA), half of the vines were treated with 5 ppm CPPU (Cruz-Castillo et al., 2014) for $10 \mathrm{~s}$. Similar fruit growing on another 20 vines were treated with water only as the control group. During the experiment, the orchard received normal agricultural practices, and no extreme weather conditions were recorded. Kiwifruit samples were collected at 25, 40, 55, 70, 85, 100, 115 and 130 DAA. At each sampling date, 10 randomly selected fruits were harvested from six vines for per treatment and there were three replications.

Fruit samples were collected from three biological replicates, snap-frozen in liquid nitrogen, and then stored at $-80^{\circ} \mathrm{C}$ for subsequent analyses.

\section{Evaluation of fruit weight, dry matter, shape and SSC}

Fruit fresh weight and dry matter were measured as reported by Nardozza et al. (2010) Shape traits (length and minimum and maximum diameters) were measured using Vernier calipers. SSCs were assayed in juice extracted from both the ends of the fruit using a WYT-4 refractometer (Jingmi, Shanghai, China).

\section{Total soluble sugars, TA, soluble protein, starch and Vitamin $\mathbf{C}$ concentrations}

Total soluble sugars was extracted using the method of Irigoyen (1992). In total, $0.5 \mathrm{~g}$ of fresh pulp was ground to a powder in the presence of liquid nitrogen, and then $15 \mathrm{~mL} 80 \%$ ethanol was added. Samples were centrifuged for $10 \mathrm{~min}$ at $7000 \mathrm{rpm}$. Afterwards, $2 \mathrm{~mL}$ of supernatant were saved for the soluble sugar determination. Total soluble sugars were assessed using the colorimetric anthrone method (Huang et al., 2017). TA and soluble protein concentrations were determined as described in Kwon et al. (2019) and Wang et al. (2016), respectively. The precipitation obtained when extracting sugars was autoclaved for $1 \mathrm{~h}$ and then incubated with amyloglucosidase in $12 \mathrm{~mL}$ acetate buffer $\left(0.25 \mathrm{M}, \mathrm{pH} 4.5,55^{\circ} \mathrm{C}\right)$ for a $1 \mathrm{~h}$. The sample was then centrifuged for $10 \mathrm{~min}$ at 7,000 $\mathrm{rpm}$. The supernatant was used to measure the starch concentration using a colorimetric determination (Smith et al., 1992). Vitamin C was measured using a 2,6- 
119 dichloroindophenol titration method (Ma et al., 2017; Alhassan et al., 2019).

120

121

122

123

124

125

126

127

128

129

130

131

132

133

134

135

136

137

138

139

140

141

142

143

144

145

146

147

148

149

150

151

152

153

154

155

156

157

158

\section{HPLC analyses of organic acids and carbohydrates}

Organic acids were detected according to Zheng et al. (2009). In total, $10 \mathrm{~g}$ kiwifruit was extracted in a $50-\mathrm{mL}$ centrifuge tube containing $12.5 \mathrm{~mL} \mathrm{NH}_{4} \mathrm{H}_{2} \mathrm{PO}_{4}(40 \mathrm{mmol} / \mathrm{L}, \mathrm{pH} 2.5)$ and centrifuged at $12,000 \times \mathrm{g}$ at $4{ }^{\circ} \mathrm{C}$ for $15 \mathrm{~min}$. The supernatant was collected and subsequently filtered. An HPLC analysis was performed using an Agilent 1200 Series instrument with a C18 column $\left(4.6 \mathrm{~mm} \times 250 \mathrm{~mm}, 5 \mu \mathrm{m}\right.$; Waters, UK) at $210 \mathrm{~nm}$. The mobile phase was $\mathrm{NH}_{4} \mathrm{H}_{2} \mathrm{PO}_{4}(40$ $\mathrm{mmol} / \mathrm{L}, \mathrm{pH} 2.5$ ). The sugar concentration was calculated as described in Barboni et al. (2010) using an Agilent 1200 Series instrument equipped with a refractive index detector (Agilent, USA), and the extract was separated using an amino column (Inertsil NH2 $250 \mathrm{~mm} \times 4.6 \mathrm{~mm}, 5 \mu \mathrm{m}$ ) and eluted with water (Milli-Q) at a flow rate of $0.6 \mathrm{~mL} / \mathrm{min}$. Elution was carried out isocratically for $20 \mathrm{~min}$ at $85^{\circ} \mathrm{C}$.

\section{Data analyses}

The experimental data are presented as the means \pm standard errors from three independent experimental replications. In the analysis process, the main factor of the ANOVA is whether CPPU was added. A single factor ANOVA (ANOVA-LSD) was used to make comparisons among groups. A difference was considered statistically significant at $p<0.05$. In the early stage of data collection, each sample was repeated three times. In the analysis process, taking time as a factor, whether the fruit was treated was correlated with the change in nutritional index content within equal time periods was considered. The software R 3.0.0 (R Development Core Team, 2008) and SPSS 16.0. were mainly used for the analyses. The statistical analysis was mainly performed using time as the dependent variable. For repeated tests, the average value of three repeated test results at each time point was used in the regression analysis to reduce the error caused by the use of a single test.

\section{Results}

\section{Fruit length, diameter and weight}

The application of $5 \mathrm{ppm}$ CPPU increased A. chinensis 'Hongyang' fruit weight and size at harvest (Fig. 1a, b). There was no obvious difference in the fresh weights of CPPU-treated and control fruit from 25 to 55 DAA. However, the fresh weight was significantly increased by the CPPU treatment from 70 to 130 DAA. At harvest, CPPU-treated fruit had a final fresh weight that was, on average, $14 \%$ greater than that of the control (Fig. 1b).

Compared with untreated fruit, the CPPU-treated fruit had greater fruit lengths and maximum diameters by $8.2 \%$ and $7 \%$, respectively, at harvest (Fig. 1c, d). The length was significantly increased from 70 to 130 DAA, and the maximum diameter was enhanced from 55 DAA until the fruit was harvested. However, the minimum fruit diameter was not influenced by CPPU application during kiwifruit development (Fig. 1e).

\section{Fruit dry matter and soluble solids concentration}


The dry matter and SSC were not affected by CPPU treatments. The percentage of fruit dry matter increased during fruit development as expected (Fig. 2a). The SSC did not change from 25 to 100 DAA. However, there was a dramatic growth from 115 DAA to 130 DAA, with the maximum SSC occurring at harvest in both treated and untreated fruit (Fig. 2b).

\section{Soluble sugar, TA, Vitamin $C$, starch and protein}

The application of CPPU elevated fruit soluble sugar concentrations. Soluble sugar accumulation was low in both treated and untreated fruit from 25 to 100 DAA. After 100 DAA, there was a significant increase in the soluble sugar level. Moreover, the soluble sugar concentration in CPPU-treated fruit was greater than in untreated fruit (Fig. 3a). At harvest, CPPU applications resulted in a $\sim 14.5 \%$ increase in soluble sugars compared with the untreated fruit.

On the other hand, CPPU did not significantly affect the TA level from 25 to 100 DAA; however, the effect was obvious from 115 DAA until fruit harvest (Fig. 3b). Where, the application of CPPU led to a decline of $\sim 6.4 \%$ in the TA concentration, compared with the untreated fruit. Meanwhile, the Vitamin C, starch and soluble protein were not significantly influenced by CPPU. The Vitamin C concentration decreased (Fig. 3c), while the starch and protein concentrations increased during 'Hongyang' kiwifruit development (Fig. 3d, e).

\section{Sugars and organic acids}

CPPU applications significantly increased fructose and glucose concentrations. Differences in fructose levels between treated and untreated fruit were first detectable at 70 DAA and continued until harvest. In addition, from 85 to 130 DAA, the glucose concentration of the CPPU-treated fruit was much greater than that of the untreated fruit. By harvest time, the CPPU applications had significantly increased the fructose and glucose concentrations in fruit by $75.2 \%$ and $43.1 \%$, respectively, compared with untreated fruit (Fig. 4a, b). On the contrary, the CPPU application decreased the sucrose concentration. The effect was evident in fruit from 85 DAA until final harvest. Finally, the sucrose concentration of CPPU-treated fruit was significantly lower by approximately $23.5 \%$, compared with the concentration of untreated fruit (Fig. 4c).

The quinic acid concentration of fruit declined after CPPU applications. There was a difference in the quinic acid concentration at 70 DAA until final harvest. Finally, the CPPU application caused a decrease of $\sim 19.5 \%$ in quinic acid compared with the untreated fruit (Fig. $4 \mathrm{~d}$ ). The citric acid concentration was not significantly affected by CPPU from 25 to 115 DAA. However, at 130 DAA, the citric acid level in the control fruit was significantly greater than that of CPPU-treated fruit. When the fruit was harvested, the citric acid concentration in untreated fruit was $\sim 18 \%$ greater than that in CPPU-treated fruit (Fig. 4e). For malic acid, CPPU applications did not significantly influence the level, compared with the control fruit (Fig. 4f).

\section{The correlations between fruit weight and nutritional parameters}

For the fruit sample data, the log function was used to linearize the glucose concentration distribution and a linear regression equations of this fruit nutrient as well as the others and weight were obtained. The regression results are shown in Figure 5a-e and Table 1, and almost all the 
200 results had good degrees of fit $\left(\mathrm{R}^{2}>0.8\right)$. For sugars, there were no significant differences in the

201 slope and intercept between CPPU-treated and control fruit. Using the same statistical analysis

202 method, quinic acid and citric acid had values greater than 0.05 for both the slopes and intercepts.

203

\section{Discussion}

Fruit weight/size and dry matter are important integrated indices used to evaluate fruit quality (Jaeger et al., 2011). In this study, the fruit weight curve of 'Hongyang' kiwifruit was similar with that of 'Hayward' kiwifruit. There was a rapid development from 25 to 55 DAA (Fig. 1b), and fresh weight continued to rapidly increased in the 'Hayward' kiwifruit from 35 to 75 DAA (Nardozza et al., 2017). After CPPU application, the fresh fruit weight significantly increased (Fig. 1b). Similar results were also reported in the 'Hayward' kiwifruit after the $10 \mathrm{mg} / \mathrm{L} \mathrm{CPPU}$ treatment at 15 days after fruit set, whereas the 'Hongyang' kiwifruit weight increase was small compared with that of the 'Hayward' kiwifruit, which increased by $30 \%$ (Ainalidou et al., 2015). In contrast, the increases in the dry matter were only slightly different between the 'Hongyang' and 'Hayward' kiwifruit when 10 ppm CPPU was applied at 28 DAA (Nardozza et al., 2017). The dry matter dramatically increased from 40 to 55 DAA in 'Hongyang' kiwifruit, while the rate of increase in 'Hayward' was basically constant. At harvest, the dry matter was not affected by CPPU treatments (Fig. 2a), which agreed with the data from CPPU-treated 'Hayward' kiwifruit when 4 ppm CPPU was sprayed $\sim 1$ week before anthesis (Cruz-Castillo et al., 2014).

The fruit length and diameter define the shape of the fruit. CPPU applications promote cell division and/or expansion to increase fruit size (Lewis et al., 1996). The length curve of 'Hongyang' kiwifruit presented as single sigmoidal in shape (Fig. 1c), and it was different from that of 'Mitsuko' kiwifruit receiving the $5 \mathrm{mg} / \mathrm{L} \mathrm{CPPU}$ treatment at 10 days after petal fall, which showed a double sigmoidal curve (Kim et al., 2006). The shape of CPPU-treated 'Hongyang' kiwifruit was a little thinner at the blossom end, compared with untreated fruit (Fig. 1c, d).

$\mathrm{SSC}$ is a crucial factor for evaluating the fruit ripening time (Koutsoflini et al., 2013). No significant differences in SSC values were observed between untreated and CPPU-treated fruit (Fig. 2b). SSC refers to all compounds dissolved in water, including sugar, acid, fibrin and mineral components, although the CPPU application increased the fruit soluble sugar concentration, it did not affect the SSC. This may result from the decreases in sucrose, quinic acid, citric acid and other soluble substances. Similar results and growth patterns were observed by Iwahori et al. (1988) in $40 \mathrm{mg} / \mathrm{L}$ CPPU-treated 'Hayward' kiwifruit.

Sugars and organic acids are the parameters of greatest concern regarding the flavour of fruit (Nishiyama et al., 2008; Richardson et al 2019). Compared with kiwifruit controls, the soluble sugar concentrations increased $23 \%$ by a 20 -ppm CPPU application at 14 DAA (Famiani et al., 1999), and TA level decreased 13.2\% when 10 ppm CPPU was applied at 30 days after petal fall (Pramanick et al., 2015). However, the concentrations of soluble sugars in CPPU-treated 'Hongyang' kiwifruit increased by $\sim 8.8 \%$ and TA decreased by $4.4 \%$ compared with in untreated fruit. Thus, the effects of CPPU on the taste-related characteristics of the 'Hongyang' kiwifruit were more limited than on other kiwifruit cultivars, this may be the result of different concentrations and times of CPPU applications. 
The Vitamin $\mathrm{C}$ concentration is a preferential factor for the assessment of the quality levels of many fruit. Kiwifruit contains more ascorbic acid than oranges, strawberries, lemons and grapefruit (Ma et al., 2017). Here, there were no differences in the Vitamin C concentration between CPPU-treated and untreated fruit, which was consistent with the findings in cucumber (Qian et al., 2018). However, 5mg/L CPPU treatment at 25 days after petal fall, the Vitamin C concentration in $A$. argute kiwifruit was less, than that of untreated fruit (Kim et al., 2006).

The changes that occur in protein levels during kiwifruit development are unknown. In the present study, the protein concentration showed similar values between CPPU-treated and untreated fruit (Fig. 3e). Qian et al. (2018) also reported that the protein concentration of cucumber was not affected by CPPU.

The accumulation of starch was similar to that of the 'Hayward' kiwifruit (Nardozza et al., 2017). However, the 'Hayward' kiwifruit had its maximum starch concentration at 125 DAA, while in 'Hongyang' kiwifruit this occurred at 100 DAA. There have been contradictory results on the effects of CPPU on the starch concentrations. The CPPU treatment was reported to significantly reduce the starch concentration of 'Hayward' kiwifruit (Nardozza et al., 2017). However, it has also been reported that 20-ppm CPPU-treated 'Hayward' kiwifruit at 14 DAA had higher starch concentrations than untreated fruit (Antognozzi et al., 1996). The current data indicated that CPPU applications did not result in a significant change in the starch concentration of 'Hongyang' kiwifruit (Fig. 3d). The results were consistent with other findings in 'Hayward' kiwifruit (Cruz-Castillo et al., 2014). After the kiwifruit matures, the starch degrades into soluble sugar. However, the concentrations of starch and soluble sugar were increased in the developmental processes. In addition, the changes in starch and soluble sugar were not consistent (Nardozza et al., 2010). Moreover the soluble sugars content of CPPU-treated fruit were higher than that of control in Actinidia deliciosa, while the starch content was similar to the control at harvest (Antognozzi et al.,1996).

The most abundant sugars in 'Hongyang' kiwifruit are glucose, fructose and sucrose. Citric, quinic and malic acids are the main organic acids (Nishiyama et al., 2008). Compared with the control, at the same developmental stages, there were remarkable increases in fructose and glucose levels (Fig. 4a, b), but the sucrose concentration declined at harvest after fruit was CPPU treated (Fig. 4c). Similar results were reported in the CPPU-treated 'Hayward' kiwifruit (Nardozza et al., 2017). However, there was a difference in the accumulation of glucose between 'Hongyang' and 'Hayward' kiwifruit. The glucose concentration reached its maximum value at 50 DAA in 'Hayward' kiwifruit, while the glucose level continued to increase in 'Hongyang' kiwifruit. A similar result was reported by Richardson et al. (2011) in 'Hort16A'.

According to the regression analysis between weight and major organic acid/sugar concentrations (Fig. 5), there were no significant differences in the slopes and intercepts (Table 1) for fructose, glucose, citric acid and quinic acid between the control and CPPU-treated groups. Those findings suggested that when the 'Hongyang' kiwifruit developed to the same weight, the nutrition indices (fructose, glucose, citric acid and quinic acid) were similar between untreated and CPPU-treated fruit. Therefore, although there were some effects on TA and soluble sugars in CPPU-treated 'Hongyang' kiwifruit by comparison with untreated fruit at the same time, when the 
282 fruit was grown to the same weight, CPPU treatments did not influence the nutritional components 283 of 'Hongyang' kiwifruit.

\section{Conclusions}

A CPPU application significantly altered 'Hongyang' kiwifruit development, leading to

Ainalidou A, Karamanoli K, Menkissoglu-Spiroudi U, Diamantidis G Matsi T. 2015. CPPU treatment and pollination: their combined effect on kiwifruit growth and quality. Scientia Horticulturae 193:147-154.

Alhassan N, Golding JB, Wills RB, Bowyer MC, Pristijono P. 2019. Long term exposure to low ethylene and storage temperatures delays calyx senescence and maintains 'Afourer'mandarins and navel oranges quality. Foods 8(1):19.

Antognozzi E, Battistelli A, Famiani F, Moscatello S, Stanica F, Tombesi A. 1996. Influence of CPPU on carbohydrate accumulation and metabolism in fruits of Actinidia deliciosa (A. Chev.). Scientia Horticulturae 65:37-47.

Barboni T, Cannac M, Chiaramonti N. 2010. Effect of cold storage and ozone treatment on physicochemical parameters, soluble sugars and organic acids in Actinidia deliciosa. Food Chemistry 121:946-951.

Cruz-Castillo JG, Baldicchi A, Frioni T, Marocchi F, Moscatello S, Proietti S, Battistelli A, Famiani F. 2014. Pre-anthesis CPPU low dosage application increases 'Hayward' kiwifruit weight without affecting the other qualitative and nutritional characteristics. Food Chemistry 158:224-228.

Du D, Xu M, Wang J, Gu S, Zhu L, Hong X. 2019. Tracing internal quality and aroma of a red-fleshed kiwifruit during ripening by means of GC-MS and E-nose. RSC Advances 9(37):21164-21174.

Famiani F, Battistelli A, Moscatello S, Boco M, Antognozzi E. 1999. Thidiazuron affects fruit growth, ripening and quality of Actinidia deliciosa. Journal of Pomology and Horticultural Science 74:6.

Famiani F, Proietti P, Pilli M, Battistelli A, Moscatello S. 2007. Effects of application of Thidiazuron (TDZ), Gibberellic acid (GA3), and 2, 4-Dichlorophenoxyacetic acid (2, 4-D) on fruit size and quality of Actinidia deliciosa'Hayward'. New Zealand Journal of Crop and Horticultural Science 35(3):341-347.

FAOstat. 2018. FAO Statistics. (Accessed on 22 March 2020)

Guo J, Yuan Y, Dou P, Yue T. 2017. Multivariate statistical analysis of the polyphenolic constituents in kiwifruit juices to trace fruit varieties and geographical origins. Food Chemistry 232:552-559.

He J, Wu D, Zhang Q, Chen H, Li H, Han Q, Dong H. 2018. Efficacy and mechanism of cinnamon essential oil on inhibition of colletotrichum acutatum isolated from 'Hongyang'. Frontiers in Microbiology 9:1288. 
Henwood RJT, Wargent JJ, Black M, Heyes JA. 2018. Environmental and management factors contributing to variability in flesh colour of a red kiwifruit cultivar in Newzealand. Scientia Horticulturae 235: 21-31.

Huang H, Ge Z, Limwachiranon J, Li L, Li W, Luo Z. 2017. UV-C treatment affects browning and starch metabolism of minimally processed lily bulb. Postharvest Biology and Technology 128: 105-111.

Irigoyen JJ, Einerich DW, Sánchez-Díaz M. 1992. Water stress induced changes in concentrations of proline and total soluble sugars in nodulated alfalfa (Medicago sativd) plants. Physiologia plantarum 84(1): 55-60.

Iwahori S, Tominaga S, Yamasaki T. 1988. Stimulation of fruit growth of kiwifruit, Actinidia chinensis Planch., by N-(2-chloro-4-pyridyl)-N-phenylurea, a diphenylurea-derivative cytokinin. Scientia Horticulturae 35(1-2):109-115.

Kim JG, Takami Y, Mizugami T, Beppu K, FukudaT, Kataoka I. 2006. CPPU application on size and quality of hardy kiwifruit. Scientia Horticulturae 110:219-222.

Koutsoflini A, Gerasopoulos D, Vasilakakis M. 2013. The effects of fruit maturation, delayed storage and ethylene treatment on the incidence of low-temperature breakdown of 'Hayward'kiwifruit. Journal of the Science of Food and Agriculture 93(2): 410-414.

Kwon MC, Kim YX, Lee S, Jung ES, Singh D, Sung J, Lee CH. 2019. Comparative Metabolomics Unravel the Effect of Magnesium Oversupply on Tomato Fruit Quality and Associated Plant Metabolism. Metabolites 9:231.

Lewis DH, Burge GK, Hopping ME, Jameson PE 1996. Cytokinins and fruit development in the kiwifruit (Actinidia deliciosa). I effects of reduced pollination and CPPU application. Physiologia Plantarum 98:187-195.

Ma T, Sun X, Zhao J, You Y, Lei Y, Gao G, Zhan J. 2017. Nutrient compositions and antioxidant capacity of kiwifruit (Actinidia) and their relationship with flesh color and commercial value. Food Chemistry 218:294-304.

Nardozza S, Boldingh HL, Richardson AC, Costa G, Marsh H, MacRae EA, Clearwater MJ 2010. Variation in carbon content and size in developing fruit of Actinidia deliciosa genotypes. Functional Plant Biology 37:545-554.

Nardozza S, Boldingh HL, Wohlers MW, Gleave AP, Luo Z, Costa G and Richardson AC 2017. Exogenous cytokinin application to Actinidia chinensis var.deliciosa 'Hayward'fruit promotes fruit expansion through water uptake. Horticulture Research 4:17043.

Nishiyama I, Fukuda T, Shimohashi A, Oota T .2008. Sugar and organic acid composition in the fruit juice of different actinidia varieties. Food Science and Technology Research 14: 67-73.

Niu Q, Wang T, Li J , Yang Q, Qian M, Teng Y.2015. Effects of exogenous application of GA4+ 7 and N(2-chloro-4-pyridyl)-N'-phenylurea on induced parthenocarpy and fruit quality in Pyruspyrifolia 'Cuiguan'. Plant Growth Regulation 76:251-258.

Peppi MC, Fidelibus MW. 2008. Effects of forchlorfenuron and abscisic acid on the quality of 'Flame Seedless' grapes. HortScience 43:173-176.

Pramanick KK, Kashyap P, Kishore DK, Sharma YP. 2015. Effect of summer pruning and CPPU on yield and quality of kiwi fruit (Actinidia deliciosa). Journal of Environmental Biology 36: 351-356.

Pranamornkith T, East A, Heyes J. 2012. Influence of exogenous ethylene during refrigerated storage on storability and quality of Actinidia chinensis (cv. Hort16A). Postharvest Biology and Technology 64(1):18. 
Qian C, Ren N , Wang J, Xu Q, Chen X Qi X. 2018. Effects of exogenous application of CPPU NAA and GA4+7 on parthenocarpy and fruit quality in cucumber (cucumissativus L.). Food Chemistry 243:410-413.

R Development Core Team. 2008. R: A Language and Environment for Statistical Computing. Vienna: R Foundation for Statistical Computing.

Richardson A, Boldingh H, Kashuba P, Knight G, Ellingham D. 2019. Flowering time determines the weight and composition of Actinidia chinensis var. 'Zesy002' kiwifruit. Scientia Horticulturae 246:741-748.

Richardson AC, Boldingh HL, Mcatee PA, Kularajathevan G , Luo ZW, Atkinson RG, David KM, Burdon JN, Schaffer RJ. 2011. Fruit development of the diploid kiwifruit, Actinidia chinensis 'Hort16a'. BMC Plant Biology 11:182-182

Richardson DP, Ansell J, Drummond LN. 2018. The nutritional and health attributes of kiwifruit: a review. European Journal of Nutrition 57(8):1-18.

Smith GS, Clark CJ, Boldingh HL. 1992. Seasonal accumulation of starch by components of the kiwifruit vine. Annals of Botany 70:19-25.

Stern RA, Ben-Arie R, Neria O, Flaishman M. 2003. CPPU and BA increase fruit size of 'Royal Gala' (Malusdomestica)apple in a warm climate. The Journal of Horticultural Science and Biotechnology 78:297302.

Wang Y, Shan T, Yuan Y, Yue T. 2016. Overall quality properties of kiwifruit treated by cinnamaldehyde and citral: microbial, antioxidant capacity during cold storage. Journal of Food Science 81(12):H3043-H3051.

Zhang JY, Pan DL, Jia ZH, Wang T, Wang G, Gu ZR. 2018. Chlorophyll, carotenoid and Vitamin C metabolism regulation in Actinidia chinensis 'hongyang' outer pericarp during fruit development. PLOS ONE 13:e0194835.

Zheng YJ, Duan YT, Zhang YF, Pan QH, Li JM, Huang WD. 2009. Determination of organic acids in red wine and must on only one rp-lc-column directly after sample dilution and filtration. Chromatographia 69:1391-1395. 


\section{Figure 1}

Effects of CPPU on the size (a), fresh weight (b), length (c), maximum diameter (d), minimum diameter (e) of 'Honyang' Kiwifruit.

CK: Control; CPPU: CPPU-treated fruit; DAA: days after anthesis. Each value represents the mean of three biological replicates of 10 fruit each, $* p<0.05, * * p<0.01$.

(a)



(b)

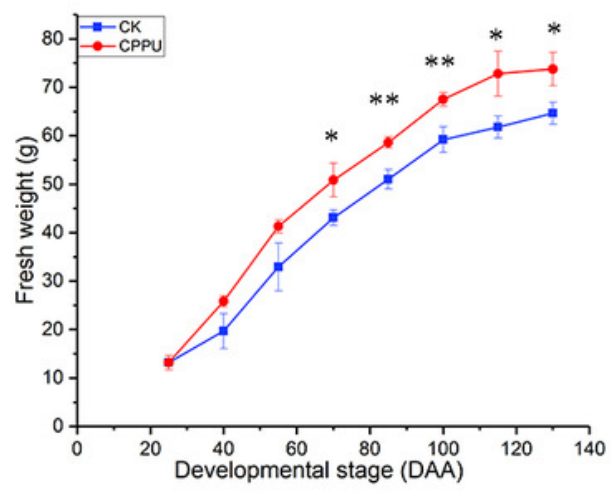

(e) (c)

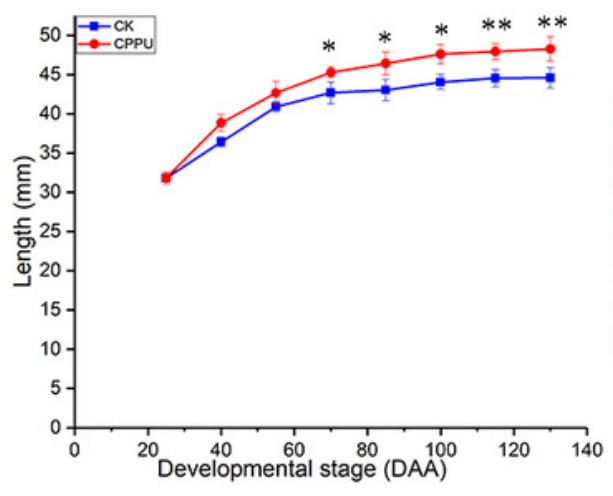

(d)

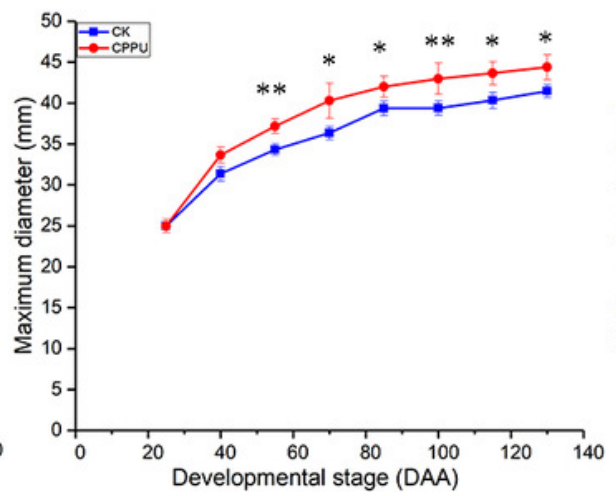

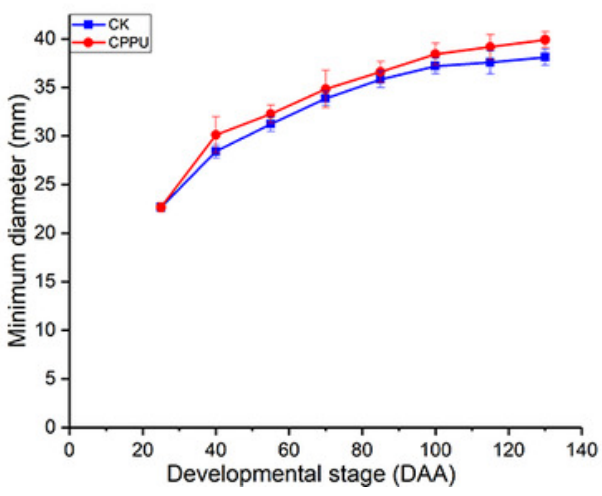


Figure 2

Effects of CPPU on the dry matter (a) and soluble solid (b) of 'Hongyang' kiwifruit.

CK: Control; CPPU: CPPU-treated fruit; DAA: days after anthesis. Each value represents the mean of three biological replicates of 10 fruit each, ${ }^{*} p<0.05, * * p<0.01$.

(a)

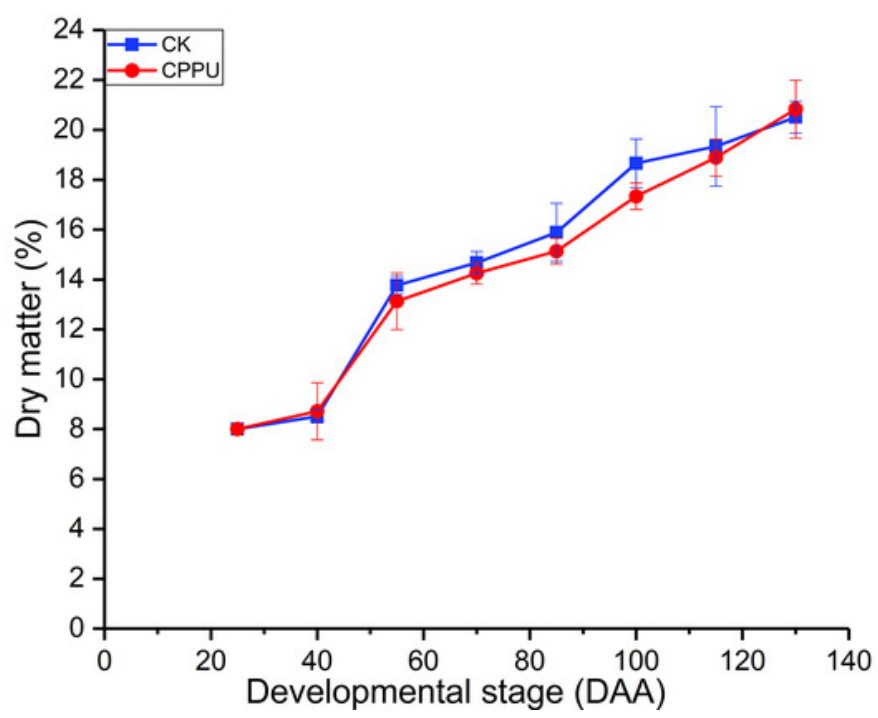

(b)




Figure 3

Effects of CPPU on the soluble sugar (a), titratable acid (b), ascorbic acid (c), starch concentration (d) and protein concentration (e) of 'Hongyang' kiwifruit.

CK: Control; CPPU: CPPU-treated fruit; DAA: days after anthesis. Each value represents the mean of three biological replicates of 10 fruit each , $* p<0.05$, ** $p<0.01$.

(a)

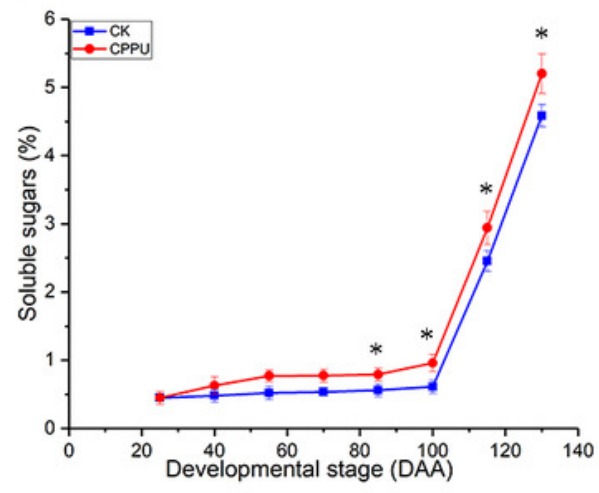

(b)

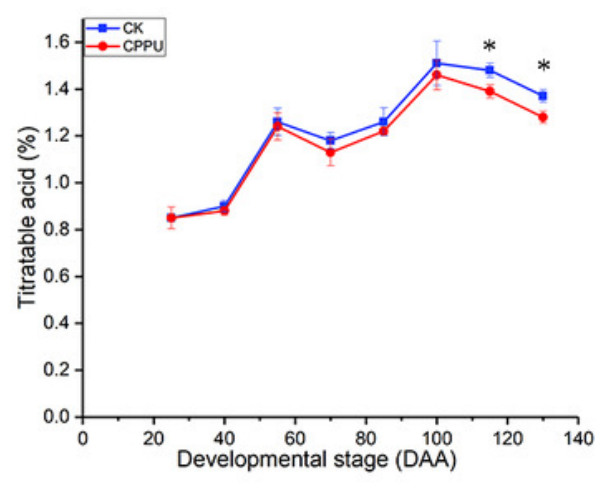

(c)

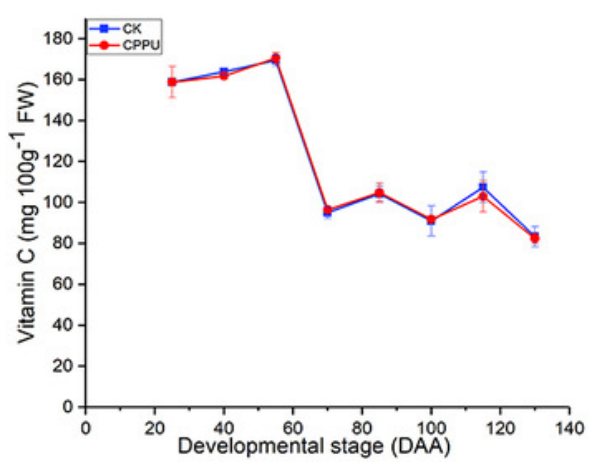

(d)

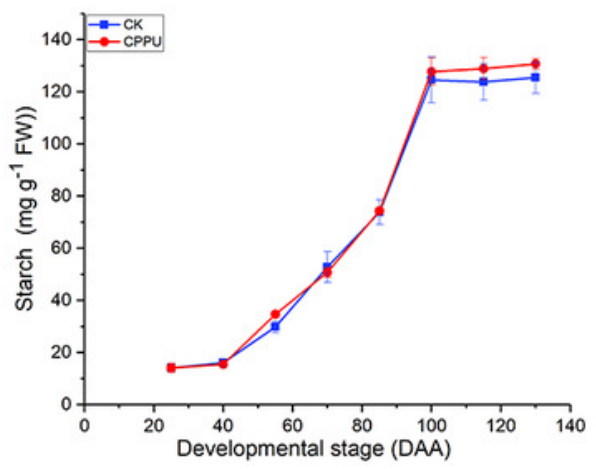

(e)

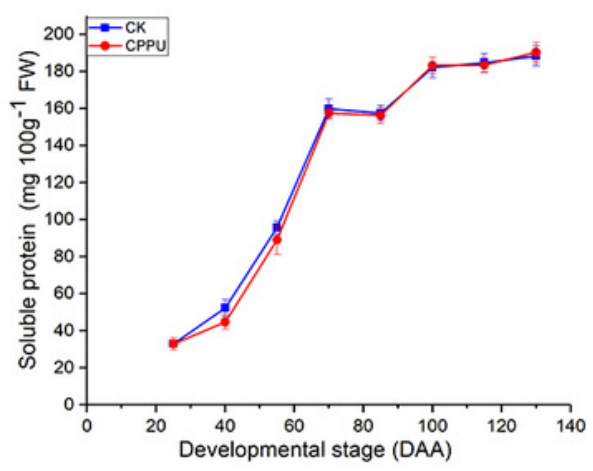


Figure 4

Effects of CPPU on the fructose (a), glucose (b), sucrose (c), quinic acid (d), citric acid (e) and malic acid (f) concentration of 'Hongyang' kiwifruit.

CK: Control; CPPU: CPPU-treated fruit; DAA: days after anthesis. Each value represents the mean of three biological replicates of 10 fruit each, $* p<0.05, * * p<0.01$.

(a)



(d)

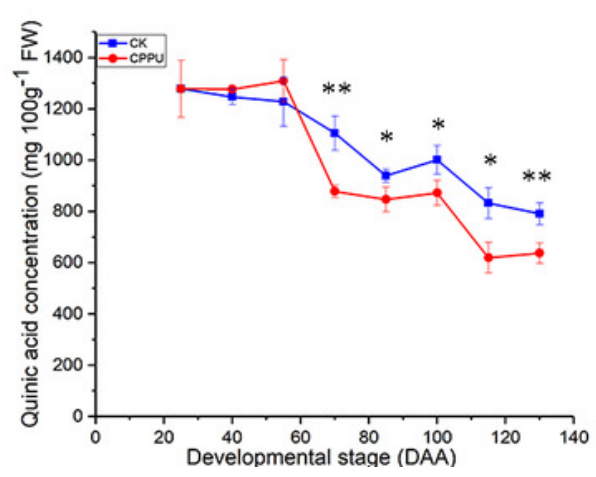

(b)

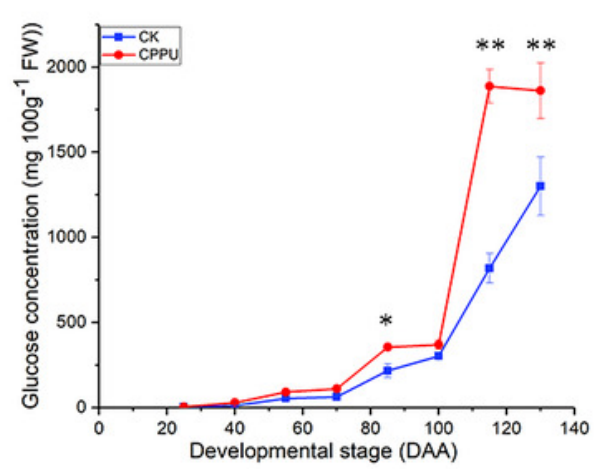

(e)

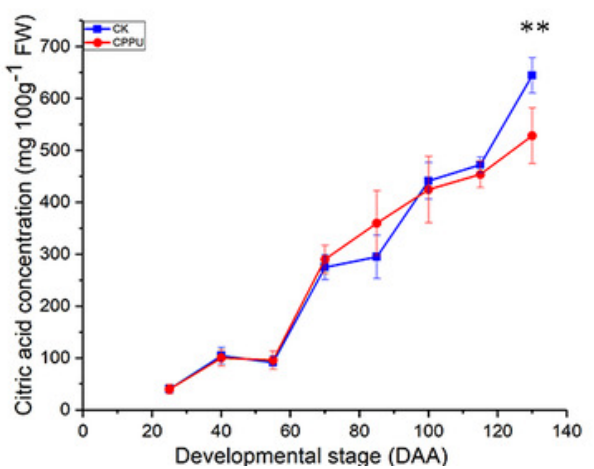

(c)



(f)

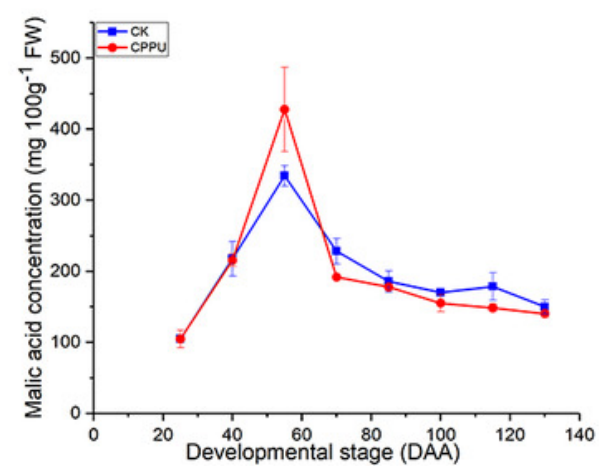


Figure 5

Regression analysis between weight and the quinic acid (a), citric (b), fructose (c), glucose (d) and sucrose (e) concentration of 'Hongyang' kiwifruit.

CK: Control; CPPU: CPPU-treated fruit.

(a)

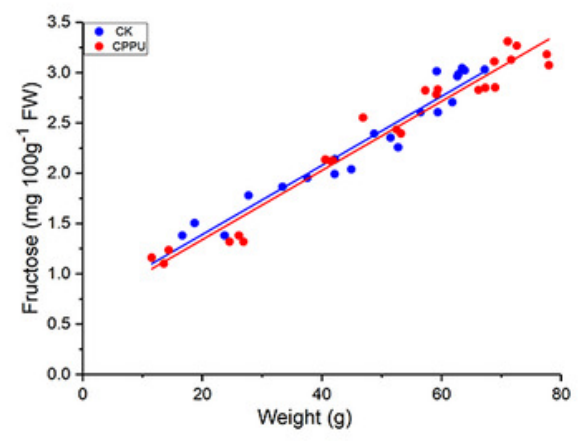

(c)

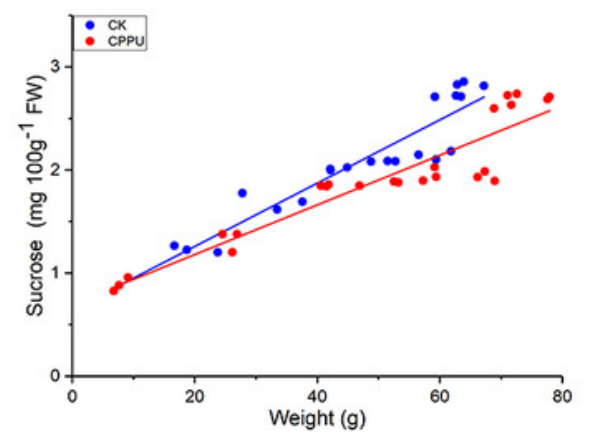

(b)

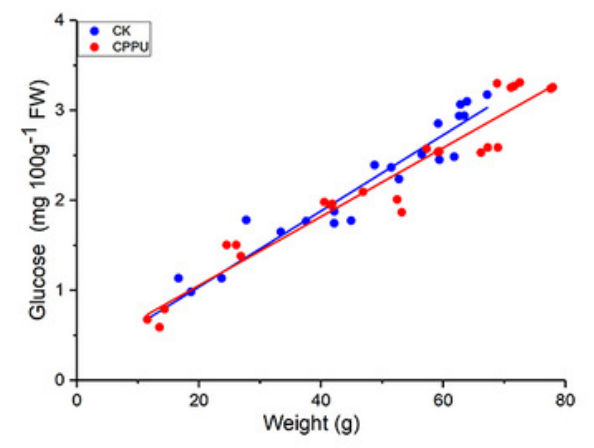

(e)






\section{Table 1 (on next page)}

Weight and the nutritional parameters regression analysis for CPPU-treated and control group fruit.

CK: Control; CPPU: CPPU-treated fruit. 


\begin{tabular}{|c|c|c|c|c|c|}
\hline & \multirow[t]{2}{*}{ Group } & \multicolumn{2}{|c|}{ Model } & \multicolumn{2}{|c|}{ Model Comparisons (P-values) } \\
\hline & & Equation & $\mathrm{R}^{2}$ & Slope & Intercept \\
\hline \multirow[t]{2}{*}{ glucose } & $\mathrm{CK}$ & $Y=0.0423 x+0.2029$ & 0.965 & 0.526 & 0.828 \\
\hline & CPPU & $Y=0.0386 x+0.2787$ & 0.954 & & \\
\hline \multirow[t]{2}{*}{ fructose } & $\mathrm{CK}$ & $Y=0.0343 x+0.7134$ & 0.974 & 0.915 & 0.694 \\
\hline & CPPU & $Y=0.0348 x+0.6323$ & 0.978 & & \\
\hline \multirow[t]{2}{*}{ sucrose } & $\mathrm{CK}$ & $Y=0.0308 x+0.6421$ & 0.961 & 0.021 & 0.887 \\
\hline & CPPU & $Y=0.0241 x+0.6982$ & 0.907 & & \\
\hline \multirow[t]{2}{*}{ Quinic acid } & $\mathrm{CK}$ & $Y=-0.0091 x+1.4482$ & 0.883 & 0.114 & 0.163 \\
\hline & CPPU & $Y=-0.0119 x+1.6152$ & 0.612 & & \\
\hline \multirow[t]{2}{*}{ Citric acid } & CK & $Y=0.0108 x-0.1553$ & 0.876 & 0.103 & 0.956 \\
\hline & CPPU & $Y=0.008 x-0.1174$ & 0.918 & & \\
\hline
\end{tabular}

